\title{
BRAF-Targeted Therapy in the Treatment of BRAF-Mutant High-Grade Gliomas in Adults
}

\author{
Tanner M. Johanns, MD, PhD; George Ansstas, MD; and Sonika Dahiya, MD
}

\begin{abstract}
Use of small molecule inhibitors specific to activating BRAF V600 mutations first demonstrated efficacy in metastatic melanoma. ${ }^{1}$ Recently, similar results were observed in patients with BRAF-mutated non-small cell lung cancer, leading to FDA approval in June 2017.2,3 Furthermore, the observation that combining BRAF inhibition (BRAFi) with MEK inhibition (MEKi) leads to improved efficacy and reduced resistance without increased toxicity has set the bar for any future multidrug combination. ${ }^{4,5}$ These observations, along with encouraging early-phase data in other histologies, such as papillary thyroid cancer ${ }^{6}$ and hairy cell leukemia, ${ }^{7}$ which both have high frequencies of BRAF V600E mutations, have led to increasing interest in exploring the role of BRAFi and MEKi in all BRAF-mutated cancers, regardless of histology. ${ }^{8}$ However, the lack of efficacy in BRAF-mutated colorectal cancer cautions against the agnostic generalization of results with these agents.
\end{abstract}

For primary central nervous system (CNS) tumors, increasing evidence has suggested that BRAFi therapy may be effective. Preliminary results from a phase I/II study of dabrafenib in relapsed or progressive pediatric BRAF V600E-mutated low-grade gliomas reported an $82 \%$ clinical benefit rate (stable disease or better at 6 months) among 32 evaluable subjects. ${ }^{9}$ A subsequent phase II study is underway evaluating the efficacy of combined BRAFi/MEKi in a similar patient population (ClinicalTrials.gov identifier: NCT02684058). Conversely, the efficacy of these agents in adult patients, particularly those with high-grade gliomas (HGGs; WHO grade III) or glioblastoma (GBM; WHO grade IV), which are more common in adults, remains undefined. Two recent publications in JNCCN - an earlier work by Johanns et $\mathrm{al}^{10}$ and an article by Schreck et al found elsewhere in this issue describing the successful use of combined BRAFi/MEKi therapy in adults with HGG or GBM-contribute to this growing body of literature, suggesting a therapeutic role for these agents in primary CNS disease, and raise several important clinical considerations.

\section{Incidence}

Several large retrospective studies have attempted to estimate the frequency of BRAF mutations in pediatric and adult primary CNS tumors ${ }^{11-15}$; for adult GBM, the frequency of these mutations is approximately $1 \%$ to $3 \%$. Interestingly, the only missense mutation found to date in adult or pediatric primary CNS tumors is the V600E variant. Despite the relatively low frequency of BRAF mutations, it is increased among several subsets of adult patients with GBM: approximately $15 \%$ of GBM cases in patients aged 17 to 35 years will harbor a BRAF V600E mutation. ${ }^{15}$ Remarkably, BRAF mutations in this cohort were found in similar frequency and were mutually exclusive to other common mutations in IDH1 (17\%) and H3F3A (19\%), suggesting that BRAF-mutated GBM represents a distinct subgroup. Likewise, the incidence of BRAF V600E is approximately $50 \%$ in epithelioid GBM (eGBM), a rare variant of IDH wild-type GBM recognized in the revised 2016 WHO classification. ${ }^{16}$ Of note, similar frequencies of BRAF V600E mutations are seen in anaplastic pleomorphic xanthoastrocytoma (aPXA), an entity with overlapping histologic and molecular features to eGBM. DNA methylation profiling of 64 histologically diagnosed eGBMs revealed a subset with a similar expression pattern to PXA that was enriched for BRAF V600E (positive in 80\%). ${ }^{17}$ Therefore, although the histologic distinction between eGBM and aPXA remains debatable based on morphology alone, the presence of either of these features in HGG (ie, epithelioid or PXA-like characteristics), as well as any IDH wild-type GBM, particularly in a patient aged $<35$ years, should trigger routine screening for the presence of a BRAF V600E mutation.

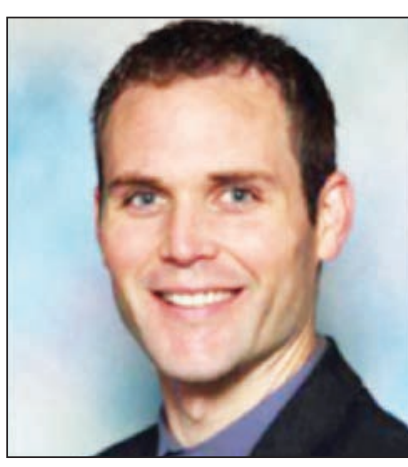

Tanner M. Johanns, MD, PhD

Tanner M. Johanns, MD, PhD, is a Clinical Fellow in the Division of Medical Oncology, Department of Medicine at Washington University School of Medicine, and is a member of the Alvin J. Siteman Cancer Center at Barnes Jewish Hospital and Washington University School of Medicine. 
Johanns et al

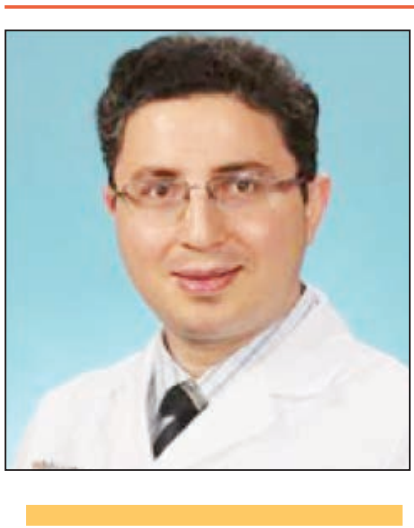

George Ansstas, MD

George Ansstas, MD, is an Assistant Professor in the Division of Medical Oncology, Department of Medicine at Washington University School of Medicine, and is a member of the Alvin J. Siteman Cancer Center and Center for Human Immunology and Immunotherapy Program at Barnes Jewish Hospital and Washington University School of Medicine.

\section{Efficacy}

Mounting evidence has suggested that BRAF-targeted therapy may be an effective treatment option for adults with BRAF-mutated HGG or GBM, but thus far has been limited to case reports. ${ }^{10,18-24}$ In total, adult HGG or GBM cases were composed of 4 GBM/eGBM, 9 aPXA, and 1 anaplastic ganglioglioma, with 10 patients treated with single-agent BRAFi (5 with vemurafenib, 5 with dabrafenib) and 4 treated with combination BRAFi/MEKi (all with dabrafenib + trametinib). With the exception of 2 patients treated in the first-line setting, all others were treated at recurrence. Together, 8 of 14 patients had not experienced progression with a median follow-up of 14 months (range, 3-27 months). Of the 6 patients who developed radiographic evidence of progression or died, median progression-free survival was 5 months (range, 1 week to 16 months) from initiation of BRAF-targeted therapy.

Despite the obvious limitations in drawing conclusions from these pooled data and small sample sizes, several important observations are worth mentioning. First, these systemically administered small molecule inhibitors appear to effectively cross the bloodtumor barrier. Moreover, 2 patients reportedly also had leptomeningeal disease and both had radiographic responses, further demonstrating adequate CNS penetration of these drugs. Of course, it remains to be determined if intracranial responses to BRAFi/MEKi are as durable as extracranial. However, in the pooled cohort of case reports, 5 patients had ongoing responses beyond 12 months. Second, many of the reports describe rapid clinical improvement after initiating BRAF-targeted therapy. ${ }^{10,19,21,23}$ This point is key given that several of the patients described had a poor performance status that precluded more aggressive treatments, such as standard cytotoxic chemotherapy or radiation. Similarly, BRAF inhibitors are generally well tolerated, with manageable side effects. Thus, they can be safely given to patients with significant functional decline. Third, most patients had received multiple lines of prior therapy, including chemotherapy and radiation, but had an impressive response rate to BRAFi therapy: 12 of 14 patients (86\%) reportedly had an investigator-assessed objective response, most of which were complete or near complete. For comparison, the intracranial response rate recently reported for combination dabrafenib/trametinib in patients with melanoma brain metastases was $56 \%$ and $58 \%$ in cohorts with or without prior local therapy, respectively. ${ }^{25}$ Together, these results provide proof-of-principle evidence that BRAF-targeted therapies in adults with high-grade CNS malignancies should be explored further.

\section{Future Perspectives}

Although the case reports discussed support the potential efficacy of BRAF-targeted therapy in adult primary CNS tumors, absent a tissue-agnostic FDA approval for BRAFtargeting agents in BRAF-mutant tumors-as was recently granted for pembrolizumab in microsatellite unstable solid tumors-larger clinical trials focused on pediatric and adult primary CNS tumors are needed to fully evaluate the efficacy and role of BRAFi and MEKi. Given the rarity of this disease, such trials will need to be performed in a multiinstitutional or international cooperative effort to allow completion in a timely manner.

Moreover, several important factors should be considered when designing a prospective study in this patient population. First, histologic inclusion criteria should be broad (ie, any adult patient with WHO grade $\geq$ III glioma with confirmed BRAF mutation) to allow for current diagnostic challenges, such as those with eGBM and aPXA discussed previously, and to accurately reflect the relevant patient population that may derive benefit from this treatment option in clinical practice. Second, leptomeningeal disease and poor performance status secondary to intracranial disease do not need to be considered exclusionary criteria. Finally, although the addition of MEKi to BRAFi may not provide any additional benefit in objective response rate compared with BRAFi monotherapy, as seen in a recent phase II study of papillary thyroid cancer, ${ }^{6}$ the lack of increased severe adverse events, reduction in secondary skin cancers, possibility of reduced resistance, and longer duration of response ${ }^{26}$ would favor the use of dual BRAFi/MEKi over single-agent regimens regardless of response rate.

In addition to defining the efficacy of BRAF-targeted therapy in primary CNS tumors, understanding the mechanisms of resistance is also clinically important. For example, al- 
though there is a subset of patients with metastatic melanoma who derive long-term benefit from BRAF-targeted therapies, ${ }^{26}$ investigators believe that resistance will ultimately develop. Yet, despite the comparatively larger experience using BRAFi and MEKi in melanoma, the exact mechanism that leads to resistance is unclear in most cases, although there are presumably multiple interrelated and unrelated pathways that can lead to the same clinical end point. ${ }^{27}$ Similarly, it would be naïve to presume that the mechanisms of drug resistance are the same in all primary BRAF-mutated CNS tumors as in extracranial disease, particularly given the inherent heterogeneity noted among the different CNS histologies alone. Therefore, pertinent questions would include whether timing, rate, or mechanism of drug resistance is the same in low-grade gliomas and HGGs, in pediatrics and adults, in primary and transformed tumors, and with single-agent BRAFi versus combination BRAFi/MEKi, or whether it is associated with specific molecular alterations and/ or immune makeup of the tumor. Thus, as more primary CNS tumors are treated with BRAF-targeted therapies, the goal should be to aggressively pursue tissue acquisition at the time of resistance to begin exploring these questions.

In conclusion, BRAF-targeted therapy represents a promising treatment option for adults with BRAF-mutated HGGs. However, work remains before these agents become standard-of-care treatment options for this patient population.

\section{References}

1. Hauschild A, Grob JJ, Demidov LV, et al. Dabrafenib in BRAF-mutated metastatic melanoma: a multicentre, open-label, phase 3 randomised controlled trial. Lancet 2012;380:358-365.

2. Planchard D, Kim TM, Mazieres J, et al. Dabrafenib in patients with BRAF(V600E)-positive advanced non-smallcell lung cancer: a single-arm, multicentre, open-label, phase 2 trial. Lancet Oncol 2016;17:642-650.

3. Planchard D, Smit EF, Groen HJ, et al. Dabrafenib plus trametinib in patients with previously untreated BRAF(V600E)-mutant metastatic non-small-cell lung cancer: an open-label, phase 2 trial. Lancet Oncol 2017;18:1307-1316

4. Long GV, Stroyakovskiy D, Gogas $\mathrm{H}$, et al. Combined BRAF and MEK inhibition versus BRAF inhibition alone in melanoma. N Engl J Med 2014;371:1877-1888.

5. Long GV, Stroyakovskiy D, Gogas H, et al. Dabrafenib and trametinib versus dabrafenib and placebo for Val600 BRAF-mutant melanoma: a multicentre, double-blind, phase 3 randomised controlled trial. Lancet 2015;386:444451.

6. Shah M, Wei L, Wirth LJ, et al. Results of randomized phase II trial of dabrafenib versus dabrafenib plus trametinib in BRAF-mutated papillary thyroid carcinoma [abstract]. J Clin Oncol 2017;35(Suppl):Abstract 6022.

7. Tiacci E, Park JH, De Carolis L, et al. Targeting mutant BRAF in relapsed or refractory hairy-cell leukemia. N Engl J Med 2015;373:1733-1747.

8. Hyman DM, Puzanov I, Subbiah V, et al. Vemurafenib in multiple nonmelanoma cancers with BRAF V600 mutations. N Engl J Med 2015;373:726-736.

9. Kieran MW, Bouffet E, Tabori U, et al. CNS tumours: the first study of dabrafenib in pediatric patients with BRAF V600-mutant relapsed or refractory low-grade gliomas [abstract]. Ann Oncol 2016;27(Suppl 6):Abstract LBA19_PR.

10. Johanns TM, Ferguson CJ, Grierson PM, et al. Rapid clinical and radiographic response with combined dabrafenib and trametinib in adults with BRAF-mutated high-grade glioma. J Natl Compr Canc Netw 2018;16:4-10.

11. Dahiya S, Emnett RJ, Haydon DH, et al. BRAF-V600E mutation in pediatric and adult glioblastoma. Neuro Oncol 2014;16:318-319.

12. Knobbe CB, Reifenberger J, Reifenberger G. Mutation analysis of the Ras pathway genes NRAS, HRAS, KRAS and BRAF in glioblastomas. Acta Neuropathol 2004;108:467-470.

13. Schindler G, Capper D, Meyer J, et al. Analysis of BRAF V600E mutation in 1,320 nervous system tumors reveals high mutation frequencies in pleomorphic xanthoastrocytoma, ganglioglioma and extra-cerebellar pilocytic astrocytoma. Acta Neuropathol 2011;121:397-405.

14. Behling F, Barrantes-Freer A, Skardelly M, et al. Frequency of BRAF V600E mutations in 969 central nervous system neoplasms. Diagn Pathol 2016;11:55.

15. Zhang RQ, Shi Z, Chen $H$, et al. Biomarker-based prognostic stratification of young adult glioblastoma. Oncotarget 2016;7:5030-5041.

16. Kleinschmidt-DeMasters BK, Aisner DL, Birks DK, et al. Epithelioid GBMs show a high percentage of BRAF V600E mutation. Am J Surg Pathol 2013;37:685-698.

17. Korshunov A, Chavez L, Sharma T, et al. Epithelioid glioblastomas stratify into established diagnostic subsets upon integrated molecular analysis [published online October 9, 2017]. Brain Pathol. doi: 10.1111/bpa.12566

18. Brown NF, Carter T, Mulholland P. Dabrafenib in BRAFV600-mutated anaplastic pleomorphic xanthoastrocytoma. CNS Oncol 2017;6:5-9.

19. Burger MC, Ronellenfitsch MW, Lorenz NI, et al. Dabrafenib in patients with recurrent, BRAF V600E mutated malignant glioma and leptomeningeal disease. Oncol Rep 2017;38:3291-3296.

20. Chamberlain MC. Salvage therapy with BRAF inhibitors for recurrent pleomorphic xanthoastrocytoma: a retrospective case series. J Neurooncol 2013;114:237-240. 
Johanns et al

21. Hofer S, Berthod G, Riklin C, et al. BRAF V600E mutation: a treatable driver mutation in pleomorphic xanthoastrocytoma (PXA). Acta Oncol 2016;55:122-123.

22. Lee EQ, Ruland S, LeBoeuf NR, et al. Successful treatment of a progressive BRAF V600E-mutated anaplastic pleomorphic xanthoastrocytoma with vemurafenib monotherapy. J Clin Oncol 2016;34:e87-e89.

23. Leaver KE, Zhang N, Ziskin JL, et al. Response of metastatic glioma to vemurafenib. Neurooncol Pract 2016;3:268-271.

24. Meletath SK, Pavlick D, Brennan T, et al. Personalized treatment for a patient with a BRAF V600E mutation using dabrafenib and a tumor treatment fields device in a high-grade glioma arising from ganglioglioma. J Natl Compr Canc Netw 2016;14:1345-1350.

25. Davies MA, Saiag P, Robert C, et al. Dabrafenib plus trametinib in patients with BRAF(V600)-mutant melanoma brain metastases (COMBI-MB): a multicentre, multicohort, open-label, phase 2 trial. Lancet Oncol 2017;18:863-873.

26. Long GV, Flaherty KT, Stroyakovskiy D, et al. Dabrafenib plus trametinib versus dabrafenib monotherapy in patients with metastatic BRAF V600E/K-mutant melanoma: long-term survival and safety analysis of a phase 3 study. Ann Oncol 2017;28:1631-1639.

27. Welsh SJ, Rizos H, Scolyer RA, et al. Resistance to combination BRAF and MEK inhibition in metastatic melanoma: where to next? Eur J Cancer 2016;62:76-85. 